\title{
Mental Health and Resilient Coping in Caregivers of Autistic Individuals during the COVID-19 Pandemic: Findings from the Families Facing COVID Study
}

\author{
Kelsey A. Friesen ${ }^{1,2}$. Jonathan A. Weiss ${ }^{3} \cdot$ Stephanie J. Howe ${ }^{1,2} \cdot$ Connor M. Kerns ${ }^{4} \cdot$ Carly A. McMorris ${ }^{1,2}$ (I)
}

Accepted: 26 June 2021 / Published online: 8 July 2021

(c) The Author(s), under exclusive licence to Springer Science+Business Media, LLC, part of Springer Nature 2021

\begin{abstract}
Many caregivers of autistic people experience mental health issues, and the impact of disruptions due to COVID-19 may present additional challenges for these individuals. This study characterized caregiver stress, anxiety, and resilient coping during COVID-19 and investigated the impact of COVID-19 disruptions, demographic variables, and resilient coping on mental health. The majority of caregivers reported some degree of disruption associated with COVID-19, and more than half reported moderate levels of stress and high anxiety. Resilient coping did not emerge as a moderator between COVID-19 disruptions and caregiver mental health, but instead had a direct effect on outcomes. Future research is needed to understand additional factors impacting the mental health of caregivers of autistic people during the COVID-19 pandemic.
\end{abstract}

Keywords Autism $\cdot$ Caregiver $\cdot$ Mental health $\cdot$ Resilience $\cdot$ COVID-19

Prior to COVID-19 it was well-established that caregivers of autistic ${ }^{1}$ individuals experience higher rates of mental health issues compared to both the general population (Baker-Ericzén et al., 2005; Bitsika \& Sharpley, 2004; Li et al., 2018) and caregivers of children with other disabilities (Dunn et al., 2001; Hayes \& Watson, 2013). These caregivers report higher levels of perceived stress (Lovell et al., 2014), depression (Taylor \& Warren, 2012), and anxiety symptoms (Ruiz-Robledillo et al., 2014), which may be associated with specific factors that accompany an autism diagnosis, including the need for assistance with daily living skills, specific behaviours (e.g. destructive, self-abusive, ritualistic, and self-stimulating), co-occurring diagnoses, as

Carly A. McMorris

camcmorr@ucalgary.ca

1 Werklund School of Education, University of Calgary, EDT 508, 2500 University Dr. NW, Calgary, AB T2N 1N4, Canada

2 Alberta Children's Hospital Research Institute, University of Calgary, Calgary, AB T2N 1N4, Canada

3 Department of Psychology, York University, 4700 Keele Street, Toronto, ON M3J 1P3, Canada

4 Department of Psychology, University of British Columbia, 2329 West Mall, Vancouver, BC V6T 1Z4, Canada well as challenges accessing supports and services (Catalano et al., 2018). Psychological distress in caregivers of autistic people also likely reflects a combination of factors, including situational stressors, child and family characteristics, and other individual factors, such as resilience. The impact of various difficulties and disruptions associated with the COVD-19 pandemic may present additional challenges over and above those typically experienced. Recently, researchers have also documented heightened COVID-19-related psychological distress in caregivers and families of autistic individuals (Ersoy et al., 2020; Esentürk, 2020; Pavlopoulou et al., 2020; Stankovic et al., 2020); however, little is known about how Canadian caregiver's mental health relates to other stressors (COVID-19 related distress) and individual factors (resilient coping) during this tumultuous time.

The Double ABCX model of family adaptation (McCubbin \& Patterson, 1983) has been used to describe the experiences of parents raising an autistic child (McStay et al., 2014), and proposes that the process of adaptation to a stressful event/situation by caregivers (e.g. COVID-19) is the product of the interaction between four factors: family demands (e.g. the accumulation of stressors, for example, child characteristics and financial strain), family adaptive

\footnotetext{
1 This paper uses the terms autistic and person with autism interchangeably, to acknowledge and respect both person-first and identity-first language.
} 
resources (e.g. social support/resources), family definition of the stressful situation (e.g. perceptions of the stressor, accumulated strains, and resources), and family adaptive coping. The mental health of caregivers of autistic individuals during COVID-19 likely represents the accumulation of COVID-19-related stressors, child characteristics, resources, appraisals, and resilience.

The impact of various disruptions linked to COVID-19 has the potential to present considerable additional challenges for parents and other caregivers. Traditional in-person social, educational, and therapeutic programs and services have been paused or significantly modified (e.g. transitioned to online platforms), and disruptions in routines and supports as a result of COVID-19 may lead to an increase in the frequency and severity of difficulties experienced by autistic individuals and their families (Eshraghi et al., 2020). As a result of physical distancing restrictions, many caregivers have adopted multiple roles (e.g. parent, educator); caregivers who have transitioned to working from home must establish a balance between caregiving and work, and those that continued or have resumed work outside of the home may be working in environments that increase their levels of personal risk (e.g. hospitals, grocery stores). These factors have the potential to contribute to higher levels of stress (Griffith, 2020) in an already stressed group of caregivers. Living through a pandemic has also been associated with feelings of ambiguity, uncertainty, and loss of control, all of which may further contribute to stress and distress (Shanahan et al., 2020). Demands placed on caregivers of autistic individuals as a result of physical distancing policies may result in caregivers experiencing magnified psychological distress (e.g. stress, distress, anxiety), as well as safety-related anxieties (e.g. about protecting their child), due to increased caregiving demands and a loss of support (Lee et al., 2020).

To date only a few studies (Ersoy et al., 2020; Esentürk, 2020; Mannging et al., 2020; Pavlopoulou et al., 2020; Stankovic et al., 2020; White et al., 2021), to our knowledge, have described the mental health and well-being of caregivers of autistic people during the pandemic. Manning and colleagues (2020) investigated the COVID-related experiences of 471 autistic individuals and their families in the United States using an online survey. Caregivers of younger individuals and those with greater severity of autism traits reported higher levels of stress. Stress was particularly associated with the disruption of therapeutic services, isolation, illness, and finance. Similarly, in a survey of 3502 parents and caregivers of autistic individuals, White and colleagues (2021) found that the majority of respondents reported moderate to extreme levels of stress, and close to $50 \%$ endorsed moderate/marked levels of family distress.

Family and caregiver outcomes are further impacted by the accumulation of child and family demands, along with COVID-specific demands. Child characteristics, such as high activity level, low mood, high support needs (Konstantareas \& Papageorgio, 2006; Zhou et al., 2019), noncompliant behaviour (Lecavalier et al., 2006), disruptive behaviour, social disability, low adaptive functioning (Postorino et al., 2019), intellectual disability, and emotional and behavioural difficulties (Kerns et al., 2015; Salomone et al., 2018) have been correlated with parent reports of distress, mental health difficulties, and quality of life. Parental factors can also emerge as contributing factors, such as maternal age (Falk et al., 2014), low levels of perceived social support (Gray \& Holden, 1992; Marsack \& Samuel, 2017), high caregiver educational levels, and low household income (Salomone et al., 2018). Many autistic children and youth require various supports, and families often also face barriers in accessing and receiving the care they need (Zablotsky et al., 2014). In addition, healthcare expenses and reduced employment may further contribute to financial strain for caregivers (Hoefman et al., 2014; Li et al., 2018).

Resilient coping, or the management of a stressful situation that leads to an adaptive or positive outcome, can aid caregivers in managing the stressors associated with caring for an autistic individual (Behket et al., 2012). For the purposes of the present study resilient coping can be operationalized as the actions that an individual may take to manage stressors in an adaptive way, leading to a positive outcome. Compared to the general population, caregivers of autistic individuals report low resilient coping, which is suggested to buffer against anxiety and depression in caregivers of autistic and non-autistic people (Bitsika et al., 2013). Consistent with this, one study found that caregivers of autistic individuals that are high in self-efficacy, optimism, and acceptance (indicators of resilient coping) are better able to cope with the challenges associated with caring for an autistic individual (Bekhet et al., 2012), and links have been found between resilient coping and mental and physical health outcomes. Specifically, caregivers who are high in resilient coping have increased marital quality, life satisfaction, and quality of life, as well as less depression, anxiety, insomnia, somatic symptoms, and parenting stress (Bayat, 2007; Bekhet et al., 2012; Ruiz-Robledillo et al., 2014).

In the context of COVID-19, researchers have highlighted that caregivers of autistic individuals are having difficulty managing demands (White et al., 2021); however, the role of individual factors in caregiver's experience during COVID19, such as resilient coping, has not been investigated. In particular, it is unknown whether caregiver stress and anxiety during the pandemic is explained by an accumulation of COVID-19 related stressors, the presence of particular child and caregiver demographic variables, and a lack of resources. Further, it is unclear how resilient coping may impact the association between COVID-19-related stresses and caregiver mental health; that is, whether it acts as a moderating factor, or a buffer, between COVID-19 difficulties 
and disruptions and caregiver stress and anxiety, or simply as an independent contributor, directly associated with caregiver mental health alongside the collective impact of stressors and other individual factors.

It is vital that caregiver mental health during and following COVID-19 is better understood and supported, as caregiver well-being has long-lasting implications for both the caregivers themselves, as well as their children and family units (Prime et al., 2020; Russell, 2020). Thus, the present study: (1) characterized caregiver stress (one's perceptions of their current situation as stressful, i.e. unpredictable, uncontrollable, and overloading), state anxiety (presence of current symptoms of anxiety, including feelings of tension and worry), and resilient coping (management of a stressful situation resulting in an adaptive outcome) during COVID-19; (2) identified the cumulative association of demographic factors (i.e. child and family characteristics), other individual factors (e.g. resilient coping), and COVID19 disruptions associated with caregiver stress and anxiety; and (3) determined if caregiver's resilient coping moderates the association between COVID-19 disruptions and caregiver mental health. Consistent with previous research, we expected that caregivers of autistic children would report high levels of perceived stress and anxiety and low levels of resilient coping. It was also anticipated that the cumulation of various demographic variables (e.g. high number of autistic children in the home, low family financial management, etc.), difficulties and disruptions associated with COVID-19, and individual variables (e.g. low resilient coping) would be associated with higher caregiver stress and anxiety. It was also expected that the association between difficulties and disruptions due to COVID-19 and caregiver's stress and anxiety would be moderated by caregiver's degree of resilient coping.

\section{Methods}

\section{Participants and Procedure}

\section{Participants}

616 Canadian caregivers of autistic individuals participated in the Families Facing COVID study (59.3\% female). The largest proportion of respondents resided in Ontario (36.1\%), British Columbia (25\%), and Alberta (20.2\%). The majority of respondents were married or common law $(89.1 \%)$ and reported living in suburban or urban communities (86.7\%). Only one caregiver per family participated, and respondents were given the option to complete the survey for up to two children. The largest proportion of respondents (74.4\%) indicated that prior to COVID-19 their family was managing financially, while a smaller group (25.6\%) reported financial difficulties. The majority of caregivers (94.3\%) reported having one autistic child in the household, most of whom were male $(66.1 \%)$, with the largest proportion being between 1 and 10 years of age $(79.2 \%, M$ age $=8.7$ years, $S D=4.2)$. Approximately $43.2 \%$ of respondents described the severity of their child's autism traits as mild, $45.5 \%$ as moderate, and $11.4 \%$ as severe. $45.1 \%$ of respondents indicated that their child experienced mental health issues prior to COVID-19.

\section{Measures}

\section{Demographic Information}

Respondents were asked to provide basic demographic information about their autistic child (e.g. gender, diagnoses), their family (e.g. number of people living in the household) and the participating caregiver (e.g. gender, age, marital status). Caregivers were also asked how they were managing financially prior to COVID-19 (i.e. We manage very well, We manage quite well, We manage, We have some trouble managing, We don't manage very well/have some financial difficulties, We are in deep financial trouble). Information regarding caregiver ethnicity, income, and education were not collected.

\section{Disruptions and Difficulties}

Participants were asked to provide Likert-type scale ratings indicting the degree to which a series of statements applied to their family. Respondents rated seven items, listed in Table 1, according to the following scale: $0=$ Not at all, 1=Somewhat, $2=$ Moderately, $3=$ Quite a bit, $4=$ Extremely. A total score ranging from 0 to 28 was created by adding the scores for each item.

\section{Perceived Stress Scale (PSS-10; Cohen \& Williamson, 1988)}

This 10-item scale is a self-report questionnaire designed to measure the degree to which individuals perceive situations in their lives as stressful and asks participants to rate how often they have experienced each statement in the past month on a 5-point Likert-type scale from 0 (Never) to 4 (Often). For example, How often have you been upset because of something that happened unexpectedly? Total scores were calculated by adding up the scores for each item, and ranged from 0 to 40 , with higher scores indicating higher perceived stress. Consistent with previous research (AlAteeq et al., 2021; Alharbi \& Alshehry, 2019; Toto \& Limone, 2021; Wiriyakijja et al., 2020), a total score between 0 and 13 was considered to be low stress, 14 to 26 , considered to be moderate stress, and scores 27 and above indicated high perceived stress. The psychometric properties of the three versions of the PSS (PSS-14, PSS10 , and PSS-4) have been examined in various populations 
Table 1 Disruptions and difficulties experienced by caregivers as a result of COVID-19 (\% of participants)

\begin{tabular}{|c|c|c|c|}
\hline & Not at All & $\begin{array}{l}\text { Somewhat/Mod- } \\
\text { erately }\end{array}$ & $\begin{array}{l}\text { Quite a bit/ } \\
\text { Extremely }\end{array}$ \\
\hline To what extent has your family's life been disrupted by the COVID-19 pandemic? & 2.4 & 44.5 & 53.1 \\
\hline Has your family experienced financial hardship? & 11.5 & 53.4 & 35 \\
\hline Have you feared for your own or your family's health or safety? & 7.6 & 52.3 & 40.1 \\
\hline Have your children feared for their own or their family's health or safety? & 10.2 & 49 & 40.8 \\
\hline Has your family had difficulty getting food? & 24.2 & 50.5 & 25.3 \\
\hline $\begin{array}{l}\text { Has your family experienced a shortage of essential goods (e.g. toilet paper, cleaning } \\
\text { supplies) }\end{array}$ & 16.9 & 59.5 & 23.7 \\
\hline Has your family had difficulties finding childcare? & 13.5 & 48.4 & 38.2 \\
\hline
\end{tabular}

$N=616$

(e.g. general population, adults, college/postgraduate students, psychiatry patients, women, adults, etc.). In a review evaluating the psychometric properties of the PSS-10, the Cronbach's alpha was $>0.70$ in 12 studies, and test-retest reliability met the criterion of $>0.70$ in four studies (Lee, 2012). Internal consistency for the PSS-10 was acceptable in the present sample (Cronbach's alpha $=0.62$ ).

\section{Short-form of the State-Trait Anxiety Inventory (STAI-6; Marteau \& Bekker, 1992)}

Caregiver level of state anxiety (i.e. how an individual is feeling in the moment) was measured using the six-item short-form version of the STAI (STAI-6), which has been shown to be highly correlated with the full 20-item version (internal consistency reliabilities $>0.90$ ). The STAI-6 prompts participants to respond to six different items according to a 4-point Likert-type scale from 1 (Not at all) to 4 (Very much): I feel calm, I am tense, I feel upset, I am relaxed, I feel content, and I am worried. Items are reverse scored so that higher scores represent higher levels of anxiety, with total scores that range from 6 to 24. Similar to previous studies (Wong et al., 2020), total scores were prorated (i.e. multiplied by 20/6) to obtain scores equivalent to the full 20-item STAI, which ranges from 20 to 80 , and similar to previous studies, were classified as no or low anxiety (20-37), moderate anxiety (38-44), and high anxiety (45-80). In a sample of parents with infants, the STAI-6 showed favourable internal consistency (Cronbach's alpha $>0.90$ ) and validity when correlated with the 20-item version (Tluczek et al., 2009). More recently, the STAI-6 was used to measure anxiety symptoms in residents of Taiwan during COVID-19 (Wong et al., 2020).

\section{The Brief Resilient Coping Scale (BRCS; Sinclair \& Wallston, 2004)}

Caregiver resilienct coping was measured using the BRCS which captures caregiver's tendencies to cope with stress in an adaptive manner. It was comprised of four items (e.g. I look for creative ways to alter difficult situations) that were rated on a five-point Likert-type scale ranging from 1 (Does not describe me at all) to 5 (Describes me very well). A total score was created by summing the ratings of each item (ranging from 4 to 20), with scores between 4 and 13 characterized as low resilience, between 14 and 16 as medium resilience, and scores over 17 as high resilience (Sinclair \& Wallston, 2004). A number of studies have used the BRCS to measure resilient coping, including caregivers of autistic children (Ruiz-Robledillo et al, 2014). The BRCS also demonstrates adequate internal consistency $(r=0.76)$ and test-retest reliability $(r=0.71)$ in the general population (Kocalevent et al., 2017).

Procedure. Participants were recruited through social media (e.g. Twitter, Instagram, Facebook, etc.) and autism agencies across Canada. Study data collection began on June $9^{\text {th }}, 2020$ and ended on July $30^{\text {th }}, 2020$. Respondents were asked to complete a short (15-20 min) online survey delivered through the [affiliation's] survey tool (Qualtrics).

\section{Data Analyses}

Data were analyzed using IBM SPSS Statistics Version 26. Prior to data analysis, a total of 634 respondents participated in the Families Facing COVID survey, of which 18 extreme outliers were removed to ensure that the assumptions of the various statistical tests were met. More specifically, due to the online nature of the survey and recruitment method the decision to remove outliers (e.g. non-sensical responses, extremely high/low scores) was made to ensure valid responses. An alpha $=0.05$ was applied to all statistical 
Table 2 Spearman's correlations among demographic factors, COVID-19 difficulties and disruptions, and caregiver stress and anxiety

\begin{tabular}{|c|c|c|c|c|c|c|c|c|c|c|c|}
\hline Variable & 1 & 2 & 3 & 4 & 5 & 6 & 7 & 8 & 9 & 10 & 11 \\
\hline \multicolumn{12}{|l|}{ Outcomes } \\
\hline Perceived stress & - & & & & & & & & & & \\
\hline State anxiety & $.47 * *$ & - & & & & & & & & & \\
\hline \multicolumn{12}{|l|}{ Contributing Factors } \\
\hline Caregiver gender & $-.13 * *$ & $-.13 * *$ & - & & & & & & & & \\
\hline Caregiver marital status & $.10^{*}$ & .08 & $.13 * *$ & - & & & & & & & \\
\hline Financial management prior to COVID-19 & $.19 * *$ & $.19 * *$ & .02 & $.09^{*}$ & - & & & & & & \\
\hline Community & .02 & -.01 & .03 & $.14 * *$ & .00 & - & & & & & \\
\hline Number of autistic children in the home & $.13 * *$ & .08 & $.13 * *$ & $.19 * *$ & -.02 & $.09 *$ & - & & & & \\
\hline Child gender & -.07 & $-.11 * *$ & -.04 & .01 & $.10^{*}$ & -.01 & .02 & - & & & \\
\hline Child mental health concerns pre-COVID-19 & $.13 * *$ & $.13 * *$ & .07 & -.01 & .04 & -.05 & -.01 & -.04 & - & & \\
\hline Difficulties and disruptions associated with COVID-19 & $.20 * *$ & .05 & $-.15^{* *}$ & .00 & $.16^{* *}$ & .03 & -.07 & .06 & $.09 *$ & & - \\
\hline Resilient coping & $-.10 *$ & $-.18^{* *}$ & -.07 & .07 & $-.11^{* *}$ & -.02 & -.06 & -.06 & -.00 & $.31 * *$ & - \\
\hline
\end{tabular}

$* p<.05, * * p<.001$

tests, unless otherwise noted. To characterize caregiver stress, anxiety, and resilient coping during COVID-19 (Aim 1), descriptive and correlational statistics were calculated. Bivariate correlational analyses and hierarchical regressions were conducted to examine the cumulative influences of demographic factors, difficulties and disruptions associated with COVID-19, and resilient coping on caregiver stress and anxiety (Aim 2). For both stress and anxiety, demographic factors were entered as independent variables in the first model, followed by difficulties and disruptions associated with COVID-19 in the second model, and caregiver resilient coping in the third model. To test for the moderation effect of resilient coping in the association between COVID-19 difficulties and disruptions and caregiver mental health (stress and anxiety), a two-way interaction term-difficulties and disruptions $x$ resilient coping (D\&D x R) - was entered into the fourth and final model. Moderation was established if there was a significant change in the $R^{2}$ from Model 3 to Model 4. To minimize multicollinearity, continuous predictor variables (i.e. difficulties and disruptions and resilient coping) were centered prior to the calculation of the two-way interaction term.

\section{Results}

The first objective of the present study was to characterize caregiver mental health during COVID-19. Overall, caregivers in the current sample were moderately stressed ( $M$ total score $=20.1, S D=4.7$ ); the majority of caregivers $(85.2 \%)$ fell into the moderate stress group, with the remainder reporting low stress (6.7\%) and high stress $(8.1 \%)$. In addition, caregivers in the present study reported high levels of anxiety ( $M$ total score $=14.8$,
$S D=3.0$ ), with $67.7 \%$ falling in the high anxiety range, $20.8 \%$ in the moderate anxiety range, and $11.5 \%$ in the no or low anxiety range. Regarding caregiver resilient coping, respondents in the present sample indicated a mean BRCS total score of $13.7(S D=2.8)$. Almost half of the sample (48.9\%) fell into the low resilience group (48.9\%), with medium resilience emerging as the second largest group (32.6\%), followed by high resilience (18.5\%). When asked about difficulties and disruptions experienced as a result of COVID-19, respondents in the present study reported a mean total score of $14.1(S D=5.1)$.

The second objective aimed to identify factors associated with caregiver stress and anxiety (Table 2 ). Caregiver gender $(p=0.001)$, and caregiver marital status $(p=0.01)$ emerged as demographic factors significantly correlated with perceived stress. Identifying as female and not being married or common law was weakly associated with higher perceived stress in caregivers. Regarding child demographic factors, the presence of mental health concerns in the child prior to COVID-19 was positively correlated with caregiver perceived stress $(p=0.001)$, though the strength of this association was weak. Finally, difficulties managing finances prior to COVID-19 $(p<0.001)$ and the number of autistic children in the home $(p=0.002)$ were associated with increased caregiver perceived stress. Total scores on ratings of disruptions and difficulties associated with COVID-19 were also significantly positively related to caregiver stress $(p<0.001)$.

Caregiver gender $(p=0.002)$ and financial management prior to COVID-19 $(p<0.001)$ emerged as caregiver factors weakly associated with anxiety, with identifying as female and financial difficulties associated with increased levels. Child gender $(p=0.008)$ and the presence of mental health concerns in the child prior to COVID-19 $(p=0.001)$ also represented relevant child factors related to anxiety, with 
Table 3 Hierarchical multiple regression predicting stress from demographics, disruptions and difficulties as a result of COVID-19, and resilient coping

\begin{tabular}{|c|c|c|c|c|c|c|c|c|}
\hline \multirow[t]{3}{*}{ Variable } & \multicolumn{8}{|c|}{ Perceived Stress } \\
\hline & \multicolumn{2}{|l|}{ Model 1} & \multicolumn{2}{|l|}{ Model 2} & \multicolumn{2}{|l|}{ Model 3} & \multicolumn{2}{|l|}{ Model 4} \\
\hline & $\mathrm{B}$ & $\beta$ & $\mathrm{B}$ & $\beta$ & $\mathrm{B}$ & $\beta$ & B & $\beta$ \\
\hline Constant & 11.05 & & 10.95 & & 11.45 & & 11.66 & \\
\hline \multicolumn{9}{|l|}{ Demographic factors } \\
\hline Financial management prior to COVID-19 & 2.22 & $.21 * *$ & 1.84 & $.17 * *$ & 1.44 & $.13^{*}$ & $1.43 *$ & .13 \\
\hline Caregiver gender & 1.16 & $.12^{*}$ & 1.49 & $.16^{* *}$ & 1.45 & $.15^{* *}$ & $1.41 * *$ & .15 \\
\hline Caregiver marital status & 1.38 & $.09 *$ & 1.33 & $.09 *$ & 1.61 & $.11^{*}$ & $1.58^{*}$ & .11 \\
\hline Child mental health & 1.14 & $.12^{*}$ & .93 & $.10^{*}$ & .87 & $.09^{*}$ & $.88^{*}$ & .09 \\
\hline Number of autistic children in the home & 2.22 & $.11^{*}$ & 2.43 & $.12 *$ & 2.20 & $.11^{*}$ & $2.16^{*}$ & .11 \\
\hline $\begin{array}{l}\text { Disruptions and difficulties as a result of } \\
\text { COVID-19 (D\&D) }\end{array}$ & & & .19 & $.21 * *$ & .25 & $.28 * *$ & $.26^{* *}$ & .28 \\
\hline Resilient coping $(\mathrm{R})$ & & & & & -.33 & $-.19 * *$ & $-.32 * *$ & -.19 \\
\hline $\mathrm{D} \& \mathrm{D} \times \mathrm{R}$ & & & & & & & -.01 & -.04 \\
\hline$R^{2}$ & .109 & & .151 & & .183 & & .185 & \\
\hline$F$ & $14.86^{* *}$ & & $17.99 * *$ & & $19.42 * *$ & & $17.18^{* *}$ & \\
\hline$\Delta R^{2}$ & .109 & & .042 & & .032 & & .002 & \\
\hline$\Delta F$ & $14.86^{* *}$ & & $30.12 * *$ & & $23.93 * *$ & & 1.38 & \\
\hline
\end{tabular}

$N=616 . * p<.05, * * p<.001$

caregivers of male children and those with mental health concerns prior to COVID-19 reporting higher levels.

Hierarchical regression analyses were used to examine the cumulative impact of demographic factors and COVID-19 related difficulties and disruptions in predicting caregiver perceived stress and anxiety. Regarding caregiver stress, Model 1, which included select demographic factors (i.e. those significantly correlated with perceived stress), was significant $\left(F(5,610)=14.86, p<0.001, R^{2}=0.11\right)$. The addition of COVID-19-related disruptions and difficulties in Model 2 was also significant $(F(1,609)=17.99, p<0.001$, $\left.R^{2}=0.15\right)$, as was the inclusion of resilient coping in Model $3\left(F(1,608)=19.42, p<0.001, R^{2}=0.18\right)$.

Next, the moderating effect of resilient coping on the association between COVID-19 difficulties and disruptions and caregiver mental health was examined. To test the hypothesized moderation model, the two-way interaction term was entered into the fourth and final model of the hierarchical regression analysis. The interaction term (D\&D x R) was inputted into the final step of the hierarchical regression. As shown in Table 3, while the overall model was significant, the interaction term was not, and did not account for any additional variance in caregiver stress $(\Delta R=0.002)$.

A second hierarchical regression was conducted to determine if the inclusion of COVID-19 difficulties and disruptions, followed by the addition of resilient coping, improved the prediction of caregiver anxiety over and above select demographic factors (i.e. those correlated with state anxiety). Model 1 was significant $\left(R^{2}=0.088, F(4,611)=14.82\right.$, $p<0.001)$. However, the addition of COVID-19-related disruptions and difficulties in Model 2 did not lead to a statistically significant increase in the variance accounted for by the model $\left(F(1,610)=12.50, p=0.08, R^{2}=0.09\right)$. The inclusion of resilient coping in Model 3 was again significant $\left(F(1,609)=15.83, p<0.001, R^{2}=0.14\right)$. Finally, the moderating effect of resilient coping on the association between COVID-19-related difficulties and disruptions and anxiety was examined (Model 4). The two-way interaction term (D\&D x RC) was again entered into the fourth and final model of the hierarchical regression analysis. Overall, the model was significant; however, the interaction term (D\&D $\mathrm{x} R$ ) was not, and did not account for any additional variance in anxiety (Table 4).

\section{Discussion}

The present study offers a unique snapshot of the experiences of 616 Canadian caregivers of autistic individuals during the COVID-19 pandemic. The majority of respondents reported some degree of disruption and difficulty as a result of COVID-19. This included financial hardship, difficulty getting food and/or finding childcare, a shortage of essential goods, and both the respondent and their child's fears for their own and their family's health and safety. The current study also characterized caregiver mental health, and in particular stress and anxiety, during COVID-19, that is, caregiver perceptions of their current 
Table 4 Hierarchical multiple regression predicting anxiety from demographics, disruptions and difficulties as a result of COVID-19, and resilient coping

\begin{tabular}{|c|c|c|c|c|c|c|c|c|}
\hline \multirow[t]{3}{*}{ Variable } & \multicolumn{8}{|l|}{ Anxiety } \\
\hline & \multicolumn{2}{|l|}{ Model 1} & \multicolumn{2}{|l|}{ Model 2} & \multicolumn{2}{|l|}{ Model 3} & \multicolumn{2}{|l|}{ Model 4} \\
\hline & $\mathrm{B}$ & $\beta$ & $\mathrm{B}$ & $\beta$ & $\mathrm{B}$ & $\beta$ & $\mathrm{B}$ & $\beta$ \\
\hline Constant & 12.68 & & 12.70 & & 13.18 & & 13.18 & \\
\hline \multicolumn{9}{|l|}{ Demographic Factors } \\
\hline Financial management prior to COVID-19 & 1.32 & $.20 * *$ & 1.24 & $.18^{* *}$ & .97 & $.14^{* *}$ & .97 & $.14 * *$ \\
\hline Caregiver gender & .71 & $.12 *$ & .78 & $.13 *$ & .76 & $.13^{*}$ & .76 & $.13 *$ \\
\hline Child mental health & .81 & $.14 * *$ & .77 & $.13^{*}$ & .73 & $.12 *$ & .73 & $.12 *$ \\
\hline Child gender & -.76 & $-.12 *$ & -.77 & $-.12 *$ & -.84 & $-.14 * *$ & -.84 & $-.14 * *$ \\
\hline $\begin{array}{l}\text { Disruptions and difficulties as a result of } \\
\text { COVID-19 (D\&D) }\end{array}$ & & & .04 & .07 & .08 & $.15^{* *}$ & .08 & $.15^{*}$ \\
\hline Resilient coping (R) & & & & & -.23 & $-.22 * *$ & -.23 & $-.22 * *$ \\
\hline $\mathrm{D} \& \mathrm{D} \times \mathrm{R}$ & & & & & & & -4.90 & .00 \\
\hline$R^{2}$ & .088 & & .093 & & .135 & & .135 & \\
\hline$F$ & $14.82 * *$ & & $12.50 * *$ & & $15.83 * *$ & & $13.55 * *$ & \\
\hline$\Delta R^{2}$ & .088 & & .005 & & .042 & & .000 & \\
\hline$\Delta F$ & $14.82 * *$ & & 3.04 & & $29.53^{* *}$ & & .00 & \\
\hline
\end{tabular}

$N=616 . * p<.05, * * p<.001$

situation as stressful (i.e. unpredictable, uncontrollable, and overloading), as well as caregiver reported current symptoms of anxiety, including feelings of tension and worry. Consistent with recent COVID-related research investigating the experiences of caregivers and families of autistic individuals (Ersoy et al., 2020; Esentürk, 2020; Manning et al., 2020; Pavlopoulou et al., 2020; Stankovic et al., 2020; White et al., 2021), the majority of participants in the sample indicated that they were experiencing moderate levels of stress and high anxiety, and the majority of caregivers were low in resilient coping. Caregiver perceived stress in the present sample appears to be comparable to that of caregivers of autistic individuals prior to COVID-19 also measured using the PSS-10 (Lovell \& Wetherell, 2016; Nik Adib et al., 2019). Compared to prepandemic stress, stress of caregivers of neurotypical children (measured using the PSS-10) has increased overtime during COVID-19 (Adams et al., 2021). This pattern may suggest that while caregivers of neurotypical children are experiencing increased stress during COVID-19, caregivers of autistic individuals were already stressed before the pandemic, and are maintaining stress levels in addition to increased difficulties/disruptions. Conversely, state anxiety of the caregivers in our current sample was heightened when compared to reports using versions of the STAI (i.e. STAI and STAI-6) with caregivers of autistic individuals pre-COVID-19 (Conner et al., 2013; Pattini et al., 2019). This trend has also been observed in mothers of neurotypical children, who showed an increase in anxiety (measured with the STAI-6) during COVID-19 compared to pre-pandemic (Racine et al., 2021).

In our sample, caregiver stress and anxiety were associated with both demographic factors and the disruptions and difficulties associated with COVID-19. For example, identifying as a female caregiver, not identifying as married or common law, having a child that demonstrated mental health concerns prior to COVID-19, reporting a greater number of autistic children in the home, and poorer financial management were associated with higher levels of perceived stress. This is consistent with emerging literature showing that caregivers of children without autism are experiencing heighted levels of anxiety and depression during COVID-19 (Racine et al., 2021). Interestingly, child age, child gender, and community (i.e. suburban/urban or rural/remote) were not significantly associated with caregiver stress in the present sample. Similarly, identifying as female and/or indicating that the family was having difficult managing finances prior to COVID-19 was also associated with increased anxiety, as was having a male child and/or a child with mental health concerns prior to COVID-19. Although these associations suggest the presence of meaningful relations between child and family demographic factors and caregiver mental health, the majority of these factors were weakly correlated. These weak correlations suggest the presence of additional influential factors that fall outside the scope of the present study. For example, one of the primary indicators of parental adaptation (e.g. stress) is the family's adaptive resources, including access to resources and social support (Ruiz-Robledillo 
et al., 2014). As these factors were not included in the analyses, it is possible that auxiliary influences, such as social support, are contributing to caregivers' functioning levels prior to COVID-19. It is also possible that a variety of additional accumulating stressors and caregiver factors (e.g. selfcompassion) were impacting reported caregiver stress. In addition, although previous research on the ABCX model has demonstrated that combinations of the aforementioned predictors explain a significant degree of variance in caregiver outcomes, unexplained variance remains to be characterized, and may have further contributed to the outcomes observed in the present study (Bohadana et al., 2019).

Caregiver mental health during COVID-19 seems to also be impacted by the accumulation of stressors and reduced resilient coping. In terms of caregiver stress, as expected, the model of demographic factors and difficulties and disruptions associated with COVID-19 was significant, and the addition of resilient coping to the prediction of stress led to an increase in variance accounted by the model. More specifically, resilient coping was associated with stress in the expected direction, with those lower in resilient coping reporting higher levels of stresss. Although the model was significant, only a small amount of the variance in caregiver stress was accounted for by the independent variables. While it seems that resilient coping may play a role in perceived stress, it does not appear to be a dominant factor impacting the mental health of caregivers in our sample.

Interestingly, caregiver anxiety was not associated with difficulties and disruptions due to COVID-19. This may be due to a variety of reasons, including limited measurement. The STAI-6 was not originally designed to assess caregiver state anxiety during a pandemic, and it is possible that the items in this scale are not addressing relevant aspects of caregiver anxiety during COVID-19. Additionally, although caregivers are reporting high levels of state anxiety overall, it is possible that disruptions and difficulties due to COVID19 are uniquely associated with caregiver stress, and that factors beyond the scope of the present study are contributing to caregiver state anxiety (i.e. anxiety about an event). Though certain demographic factors are associated with caregiver anxiety (e.g. caregiver and child gender, child mental health difficulties prior to COVID-19, and financial difficulties), the addition of difficulties and disruptions associated with COVID-19 did not explain additional variance. The inclusion of resilient coping to the prediction of caregiver anxiety was significant; however, the actual amount of variance accounted for in the final model was minimal (13.5\%).

Unexpectedly, resilient coping did not emerge as a moderator in the association between COVID-19 difficulties and disruptions and caregiver mental health (i.e. stress and anxiety). Within the resilience literature, three theoretical models have provided frameworks to understand the manner in which certain factors interact with risk to impact outcomes: compensatory, protective, and challenge. The compensatory model posits that resilient coping may have a direct main effect on outcomes, while the protective factor model asserts that resilience factors moderate the effects of risk on outcomes. The lesser-studied challenge model maintains that exposure to risk promotes the development of coping mechanisms which protects against later stressors (Zimmerman et al., 2013). Results of the present study lend support for the role of resilient coping as a compensatory factor, rather than a protective factor, in the association between COVID-19 difficulties and disruptions and caregiver mental health. More specifically, caregivers who enact active and flexible steps to manage stressors in an adaptive way appear to have decreased risk of mental health problems (i.e. stress and anxiety) in general (Bayat, 2007; Bekhet et al., 2012; Ruiz-Robledillo et al., 2014) and during these uncertain times. Further, unlike risk factors, resilient coping operates in the opposite direction, promoting caregiver mental health independent of other risk (e.g. demographic factors) and protective factors. However, future research is necessary to gain a stronger understanding of the role of resilient coping during COVID-19.

Taken together, a partial picture is emerging in which the cumulative impact of stressors and other individual factors (i.e. resilient coping) is associated with well-being. These factors are only a limited snapshot of the picture of mental health in caregivers during COVID-19. For example, resilient coping has been associated with self-efficacy, coping style, social support, cognitive appraisal, locus of control, optimism, acceptance, life satisfaction, and positive family functioning in families of autistic individuals (Bekhet et al., 2012; Iacob et al., 2020) and future research is needed to investigate these relations in caregivers during COVID19. Research on the impact of COVID-19 on caregivers of autistic people suggest that these individuals and their families are experiencing increased demands and psychological distress (Lee et al., 2020). The results of the present study are consistent with this observation, as well as represent a novel attempt to identify a key factor impacting caregiver outcomes (i.e. resilient coping). The present study did not, however, include measures addressing the broad range of stressors associated with COVID-19, and additional contributing factors were beyond the scope of the current study. To better understand the unique experiences of these individuals during an unprecedented crisis, further research is necessary to identify additional contributing factors.

\section{Limitations}

Despite many strengths of the present study, there are some limitations that need to be considered. First, the measures included were not created specifically for measuring mental health and resilient coping during a pandemic, and in many 
cases the short forms were selected for brevity in order to encourage participant completion. The mental health and experiences of caregivers likely differ between the periods prior to and during COVID-19, and the measures selected in the present study may not be effectively capturing caregiver mental health and resilient coping during these unprecedented times. Second, information regarding caregiver ethnicity was inadvertently not collected and was a major oversight in the design of the current study's questionnaire. Not collecting this information prohibits an exploration of the potential moderating role of race and ethnicity, factors that are well known to impact caregiver stress and resilient coping. Third, the presence/absence of co-occurring diagnoses in the child was not included in the current analyses. As cooccurring diagnoses in the child have been associated with caregiver outcomes, including increased depressive symptoms and greater family life difficulty (Kim et al., 2016), as well as lower quality of life (Zablotsky et al., 2013), the inclusion of this information may have provided a better understanding of the association between child factors and caregiver mental health. Fourth, regional differences were also not explored in the analysis, which may have provided a clearer picture of family experiences in response to COVID19 and associated restrictions across Canada and within provinces. Fifth, the cross-sectional design of the present study does not support inferences about the nature or direction of the associations. Sixth, survey data for the present study was collected at a relatively early time point in the pandemic (i.e. June/July 2020), and it is anticipated that caregiver mental health and perceived difficulties have likely evolved in response to the continued social distancing measures and fluctuations in isolation measures. Caregiver mental health prior to COVID-19 is also not known, and the extent to which symptoms were present prior to the implementation of self-isolation and social distancing measures cannot be presumed. Lastly, the present study relied solely on single informant reports from caregivers and thus findings could be influenced by response bias/style (e.g. a tendency for some parents to respond more negatively to some questions than others).

\section{Conclusion}

Caring for an autistic child is long-lasting, and the impact of COVID-19 on the care situation is not yet well understood. The present study highlights the impact of COVID-19 on caregiver mental health, and the majority of respondents indicated they were experiencing moderate levels of stress and high anxiety. In addition, particular caregiver and child characteristics were associated with increased risk for mental health difficulties, with caregivers identifying as female, those with poor financial management, and those with children with mental health problems prior to COVID-19 at the greatest risk for both stress and anxiety. As predicted, the majority of caregivers identified as being low in resilient coping, which may place them at an increased risk of experiencing mental health challenges. Unexpectedly, resilient coping did not emerge as a moderator in the association between COVID19 difficulties and disruptions and caregiver stress and anxiety, and future research is necessary to elucidate the role of resilience during COVID-19.

There is a strong need for psychological interventions designed to alleviate the mental health consequences of COVID-19 and resulting social distancing measures, and in particular, evidence-based tele-mental health services should be made accessible to vulnerable individuals, including caregivers of autistic individuals (Reger et al., 2020). It is imperative that future research investigate other factors that may be impacting the mental health of caregivers. Longitudinal studies are needed to evaluate the relation between resilient coping and mental health as the COVID-19 pandemic continues, and to understand the impact of the duration of COVID-19-related protective and quarantine measures, as well as the evolution of caregiver experiences over time and in response to these measures.

Acknowledgments We would like to thank all the caregivers of autistic people who participated in the Families Facing COVID study.

Author Contributions KF conducted data analysis, interpreted the data, and drafted the manuscript. JW and $\mathrm{KC}$ helped design the study survey and interpret the data. SH aided in data collection and coordination of the study. CM conceived of the study, designed and coordinated the study, collected data, and helped interpret the data. All authors read and approved the final manuscript.

\section{Declarations}

Conflict of interest The authors declare that they have no conflicts of interest to disclose.

\section{References}

Adams, E. L., Smith, D., Caccavale, L. J., \& Bean, M. K. (2021). Parents are stressed! Patterns of parent stress across COVID-19. Frontiers in Psychiatry, 12, 300-310.

AlAteeq, D. A., Alotaibi, R., Al Saqer, R., Alharbi, N., Alotaibi, M., Musllet, R., \& Alraqibah, R. (2021). Caffeine consumption, intoxication, and stress among female university students: A crosssectional study. Middle East Current Psychiatry, 28(1), 1-10.

Alharbi, H., \& Alshehry, A. (2019). Perceived stress and coping strategies among ICU nurses in government tertiary hospitals in Saudi Arabia: A cross-sectional study. Annals of Saudi Medicine, 39(1), 48-55.

Baker-Ericzén, M. J., Brookman-Frazee, L., \& Stahmer, A. (2005). Stress levels and adaptability in parents of toddlers with and 
without autism spectrum disorders. Research and Practice for Persons with Severe Disabilities, 30(4), 194-204.

Bayat, M. (2007). Evidence of resilience in families of children with autism. Journal of Intellectual Disability Research, 51(9), $702-714$.

Bekhet, A. K., Johnson, N. L., \& Zauszniewski, J. A. (2012). Resilience in family members of persons with autism spectrum disorder: A review of the literature. Issues in Mental Health Nursing, 33(10), 650-656.

Bitsika, V., \& Sharpley, C. F. (2004). Stress, anxiety and depression among parents of children with autism spectrum disorder. Australian Journal of Guidance and Counselling, 14, 151-161.

Bitsika, V., Sharpley, C. F., \& Bell, R. (2013). The buffering effect of resilience upon stress, anxiety and depression in parents of a child with an autism spectrum disorder. Journal of Developmental and Physical Disabilities, 25(5), 533-543.

Bohadana, G., Morrissey, S., \& Paynter, J. (2019). Self-compassion: A novel predictor of stress and quality of life in parents of children with autism spectrum disorder. Journal of Autism and Developmental Disorders, 49(10), 4039-4052.

Catalano, D., Holloway, L., \& Mpofu, E. (2018). Mental health interventions for parent carers of children with autistic spectrum disorder: Practice guidelines from a critical interpretive synthesis (CIS) systematic review. International Journal of Environmental Research and Public Health, 15(2), 341.

Cohen, S., \& Williamson, G. (1988). Perceived stress in a probability sample of the United States. In S. Spacapan \& S. Oskamp (Eds.), The social psychologyof health: Claremont symposium on applied social psychology (pp. 31-67). Sage.

Conner, C. M., Maddox, B. B., \& White, S. W. (2013). Parents' state and trait anxiety: Relationships with anxiety severity and treatment response in adolescents with autism spectrum disorders. Journal of Autism and Developmental Disorders, 43(8), 1811-1818.

Dunn, M. E., Burbine, T., Bowers, C. A., \& Tantleff-Dunn, S. (2001). Moderators of stress in parents of children with autism. Community Mental Health Journal, 37(1), 39-52.

Ersoy, K., Altın, B., Sarıkaya, B., \& Özkardaş, O. (2020). The comparison of impact of health anxiety on dispositional hope and psychological well-being of mothers who have children diagnosed with autism and mothers who have normal children, in Covid-19 Pandemic. Social Sciences Research Journal, 9(2), 117-126.

Esentürk, O. K. (2020). Parents' perceptions on physical activity for their children with autism spectrum disorders during the novel Coronavirus outbreak. International Journal of Developmental Disabilities. https://doi.org/10.1080/20473869.2020.1769333

Eshraghi, A. A., Li, C., Alessandri, M., Messinger, D. S., Eshraghi, R. S., Mittal, R., \& Armstrong, F. D. (2020). COVID-19: Overcoming the challenges faced by individuals with autism and their families. The Lancet Psychiatry, 7(6), 481-483.

Falk, N. H., Norris, K., \& Quinn, M. G. (2014). The factors predicting stress, anxiety and depression in the parents of children with autism. Journal of Autism and Developmental Disorders, 44(12), $3185-3203$.

Gray, D. E., \& Holden, W. (1992). Psycho-social well-being among the parents of children with autism. Australia and New Zealand Journal of Developmental Disabilities, 18, 83-93.

Griffith, A. K. (2020). Parental burnout and child maltreatment during the COVID-19 pandemic. Journal of Family Violence. https://doi. org/10.1007/s10896-020-00172-2

Hayes, S. A., \& Watson, S. L. (2013). The impact of parenting stress: A meta-analysis of studies comparing the experience of parenting stress in parents of children with and without autism spectrum disorder. Journal of Autism and Developmental Disorders, 43(3), 629-642.
Hoefman, R., Payakachat, N., Van Exel, J., Kuhlthau, K., Kovacs, E., Pyne, J., et al. (2014). Caring for a child with autism spectrum disorder and parents' quality of life: Application of the CarerQoL. Journal of Autism and Developmental Disorders, 44(8), 1933-1945.

Iacob, C. I., Avram, E., Cojocaru, D., \& Podina, I. R. (2020). Resilience in familial caregivers of children with developmental disabilities: A meta-analysis. Journal of Autism and Developmental Disorders. https://doi.org/10.1007/s10803-020-04473-9

Kerns, C. M., Kendall, P. C., Zickgraf, H., Franklin, M. E., Miller, J., \& Herrington, J. (2015). Not to be overshadowed or overlooked: Functional impairments associated with comorbid anxiety disorders in youth with ASD. Behavior Therapy, 46(1), 29-39.

Kim, I., Ekas, N. V., \& Hock, R. (2016). Associations between child behavior problems, family management, and depressive symptoms for mothers of children with autism spectrum disorder. Research in Autism Spectrum Disorders, 26, 80-90.

Kocalevent, R. D., Zenger, M., Hinz, A., Klapp, B., \& Brähler, E. (2017). Resilient coping in the general population: Standardization of the brief resilient coping scale (BRCS). Health and Quality of Life Outcomes, 15(1), 1-8.

Konstantareas, M. M., \& Papageorgiou, V. (2006). Effects of temperament, symptom severity and level of functioning on maternal stress in Greek children and youth. Autism, 10, 593-607.

Lecavalier, L., Leone, S., \& Wiltz, J. (2006). The impact of behaviour problems on caregiver stress in young people with autism spectrum disorders. Journal of Intellectual Disability Research, $50(3), 172-183$.

Lee, E. H. (2012). Review of the psychometric evidence of the perceived stress scale. Asian Nursing Research, 6(4), 121-127.

Lee, V., Albaum, C., Tablon Modica, P., Ahmad, F., Gorter, J. W., Khanlou, N., McMorris, C.A., Lai, J.C., Harrison, C., Hedley, T., Johnston, P., Putterman, C., Spoelstra, M. \& Weiss, J. A. (2020). Impact of COVID-19 on the mental health and wellbeing of caregivers and families of autistic people: A rapid synthesis review. Report prepared for the Canadian Institutes of Health Research.

Li, X. S., Pinto-Martin, J. A., Thompson, A., Chittams, J., \& Kral, T. V. (2018). Weight status, diet quality, perceived stress, and functional health of caregivers of children with autism spectrum disorder. Journal for Specialists in Pediatric Nursing, 23(1), e12205.

Lovell, B., Elliot, H., Liu, C. C. S., \& Wetherell, M. A. (2014). Memory failures for everyday tasks in caregivers of children with autism. Research in Developmental Disabilities, 35(11), 3057-3061.

Lovell, B., \& Wetherell, M. A. (2016). Behaviour problems of children with ASD and perceived stress in their caregivers: The moderating role of trait emotional intelligence? Research in Autism Spectrum Disorders, 28, 1-6.

Manning, J., Billian, J., Matson, J., Allen, C., \& Soares, N. (2020). Perceptions of families of individuals with autism spectrum disorder during the COVID-19 crisis. Journal of Autism and Developmental Disorders. https://doi.org/10.1007/s10803-020-04760-5

Marsack, C. N., \& Samuel, P. S. (2017). Mediating effects of social support on quality of life for parents of adults with autism. Journal of Autism and Developmental Disorders, 47(8), 2378-2389.

Marteau, T. M., \& Bekker, H. (1992). The development of a six-item short-form of the state scale of the Spielberger State-Trait Anxiety Inventory (STAI). British Journal of Clinical Psychology, 31(3), 301-306.

McCubbin, H. I., \& Patterson, J. M. (1983). The family stress process: The double ABCX model of adjustment and adaptation. Marriage \& Family Review, 6(1-2), 7-37.

McStay, R. L., Trembath, D., \& Dissanayake, C. (2014). Stress and family quality of life in parents of children with autism spectrum disorder: Parent gender and the double ABCX model. Journal of Autism and Developmental Disorders, 44(12), 3101-3118. 
Nik Adib, N. A., Ibrahim, M. I., Ab Rahman, A., Bakar, R. S., Yahaya, N. A., Hussin, S., \& Wan Mansor, W. N. A. (2019). Perceived stress among caregivers of children with autism spectrum disorder: A state-wide study. International Journal of Environmental Research and Public Health, 16(8), 1468.

Pattini, E., Carnevali, L., Troisi, A., Matrella, G., Rollo, D., Fornari, M., \& Sgoifo, A. (2019). Psychological characteristics and physiological reactivity to acute stress in mothers of children with autism spectrum disorder. Stress and Health, 35(4), 421-431.

Pavlopoulou, G., Wood, R., \& Papadopoilos, C. (2020). Impact of Covid-19 on the experiences of parents and family carers of autistic children and youth people in the UK. UCL Research Briefing.

Postorino, V., Gillespie, S., Lecavalier, L., Smith, T., Johnson, C., Swiezy, N., Aman, M. G., McDougle, C. J., Bearss, K., Andridge, R. R., Vitiello, B., \& Scahill, L. (2019). Clinical correlates of parenting stress in children with Autism Spectrum Disorder and serious behavioral problems. Journal of Child and Family Studies, 28(8), 2069-2077.

Prime, H., Wade, M., \& Browne, D. T. (2020). Risk and resilience in family well-being during the COVID-19 pandemic. American Psychologist, 75(5), 631-643.

Racine, N., Hetherington, E., McArthur, B. A., McDonald, S., Edwards, S., Tough, S., \& Madigan, S. (2021). Maternal depression and anxiety sypmtoms before and during the COVID-19 panademic in Canada: A Longitduinal analysis. The Lancet Psychiatry. https:// doi.org/10.1016/S2215-0366(21)00074-2

Reger, M. A., Stanley, I. H., \& Joiner, T. E. (2020). Suicide mortality and coronavirus disease 2019-a perfect storm? JAMA Psychiatry. https://doi.org/10.1001/jamapsychiatry.2020.1060

Ruiz-Robledillo, N., De Andrés-García, S., Pérez-Blasco, J., GonzálezBono, E., \& Moya-Albiol, L. (2014). Highly resilient coping entails better perceived health, high social support and low morning cortisol levels in parents of children with autism spectrum disorder. Research in Developmental Disabilities, 35(3), 686-695.

Russell, B. S., Hutchinson, M., Tambling, R., Tomkunas, A. J., \& Horton, A. L. (2020). Initial challenges of caregiving during COVID19: Caregiver burden, mental health, and the parent-child relationship. Child Psychiatry \& Human Development, 51, 671-682.

Salomone, E., Leadbitter, K., Aldred, C., Barrett, B., Byford, S., Charman, T., Howlin, P., Green, J., Le Couteur, A., McConachie, H., Parr, J. R., Pickles, A., Slonims, V., \& The PACT Consortium. (2018). The association between child and family characteristics and the mental health and wellbeing of caregivers of children with autism in mid-childhood. Journal of Autism and Developmental Disorders, 48(4), 1189-1198.

Shanahan, L., Steinhoff, A., Bechtiger, L., Murray, A. L., Nivette, A., Hepp, U., Ribeaud, D., \& Eisner, M. (2020). Emotional distress in young adults during the COVID-19 pandemic: Evidence of risk and resilience from a longitudinal cohort study. Psychological Medicine. https://doi.org/10.1017/S003329172000241X

Sinclair, V. G., \& Wallston, K. A. (2004). The development and psychometric evaluation of the brief resilient coping scale. Assessment, 11(1), 94-101.
Stankovic, M., Jelena, S., Stankovis, M., Shih, A., Stanjanovic, A., $\&$ Stankovic, S. (2020). The Serbian experience of challenges of parenting children with autism spectrum disorders during the COVID-19 pandemic and the State of Emergency with the police lockdown. The Lancet Child and Adolescent Health. https://doi. org/10.2139/ssrn.3582788

Taylor, J. L., \& Warren, Z. E. (2012). Maternal depressive symptoms following autism spectrum diagnosis. Journal of Autism and Developmental Disorders, 42(7), 1411-1418.

Tluczek, A., Henriques, J. B., \& Brown, R. L. (2009). Support for the reliability and validity of a six-item state anxiety scale derived from the State-Trait Anxiety Inventory. Journal of Nursing Measurement, 17(1), 19-28.

Toto, G. A., \& Limone, P. (2021). Motivation, stress and impact of online teaching on Italian teachers during COVID-19. Computers, 10(6), 75-86.

White, L. C., Law, J. K., Daniels, A. M., Toroney, J., Vernoia, B., Xiao, S., The SPARK Consortium, Feliciano, P., \& Chung, W. K. (2021). Brief report: Impact of COVID-19 on individuals with ASD and their caregivers: A perspective from the SPARK cohort. Journal of Autism and Developmental Disorders. https://doi.org/ 10.1007/s10803-020-04816-6

Wiriyakijja, P., Porter, S., Fedele, S., Hodgson, T., McMillan, R., Shephard, M., \& Ni Riordain, R. (2020). Validation of the HADS and PSS-10 and a cross-sectional study of psychological status in patients with recurrent aphthous stomatitis. Journal of Oral Pathology \& Medicine, 49(3), 260-270.

Wong, L. P., Hung, C. C., Alias, H., \& Lee, T. S. H. (2020). Anxiety symptoms and preventive measures during the COVID-19 outbreak in Taiwan. BMC Psychiatry, 20(1), 1-9.

Zablotsky, B., Anderson, C., \& Law, P. (2013). The association between child autism symptomatology, maternal quality of life, and risk for depression. Journal of Autism and Developmental Disorders, 43(8), 1946-1955.

Zablotsky, B., Kalb, L. G., Freedman, B., Vasa, R., \& Stuart, E. A. (2014). Health care experiences and perceived financial impact among families of children with an autism spectrum disorder. Psychiatric Services, 65(3), 395-398.

Zhou, W., Liu, D., Xiong, X., \& Xu, H. (2019). Emotional problems in mothers of autistic children and their correlation with socioeconomic status and the children's core symptoms. Medicine, 98(32), e16794.

Zimmerman, M. A., Stoddard, S. A., Eisman, A. B., Caldwell, C. H., Aiyer, S. M., \& Miller, A. (2013). Adolescent resilience: Promotive factors that inform prevention. Child Development Perspectives, 7(4), 215-220.

Publisher's Note Springer Nature remains neutral with regard to jurisdictional claims in published maps and institutional affiliations. 